\title{
Measuring Stress in Health Professionals Over the Phone Using Automatic Speech Analysis During the COVID-19 Pandemic: Observational Pilot Study
}

Alexandra König ${ }^{1}$, PhD; Kevin Riviere ${ }^{2}$, MD; Nicklas Linz ${ }^{3}$, MSc; Hali Lindsay ${ }^{4}$, MSc; Julia Elbaum², MD; Roxane Fabre $^{2}$, MSc; Alexandre Derreumaux ${ }^{5}, \mathrm{MSc}$; Philippe Robert ${ }^{5}, \mathrm{MD}, \mathrm{PhD}$

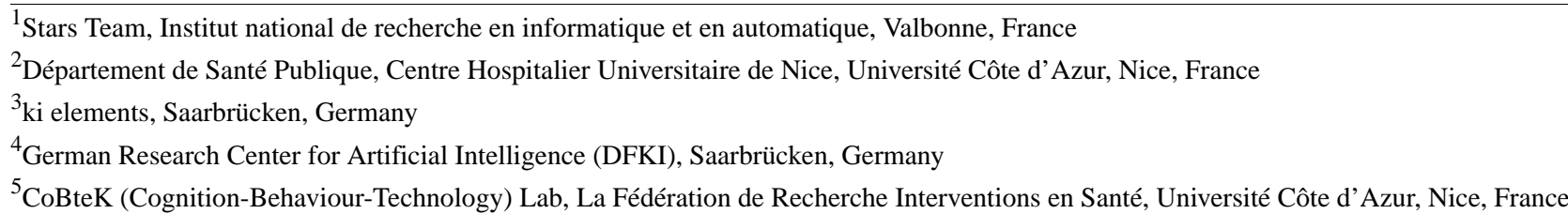

\section{Corresponding Author:}

Alexandra König, $\mathrm{PhD}$

Stars Team

Institut national de recherche en informatique et en automatique

2004 Route des Lucioles, 06902

Sophia Antipolis

Valbonne, 06200

France

Phone: $33+33652021156$

Email: alexandra.konig@inria.fr

\section{Abstract}

Background: During the COVID-19 pandemic, health professionals have been directly confronted with the suffering of patients and their families. By making them main actors in the management of this health crisis, they have been exposed to various psychosocial risks (stress, trauma, fatigue, etc). Paradoxically, stress-related symptoms are often underreported in this vulnerable population but are potentially detectable through passive monitoring of changes in speech behavior.

Objective: This study aims to investigate the use of rapid and remote measures of stress levels in health professionals working during the COVID-19 outbreak. This was done through the analysis of participants' speech behavior during a short phone call conversation and, in particular, via positive, negative, and neutral storytelling tasks.

Methods: Speech samples from 89 health care professionals were collected over the phone during positive, negative, and neutral storytelling tasks; various voice features were extracted and compared with classical stress measures via standard questionnaires. Additionally, a regression analysis was performed.

Results: Certain speech characteristics correlated with stress levels in both genders; mainly, spectral (ie, formant) features, such as the mel-frequency cepstral coefficient, and prosodic characteristics, such as the fundamental frequency, appeared to be sensitive to stress. Overall, for both male and female participants, using vocal features from the positive tasks for regression yielded the most accurate prediction results of stress scores (mean absolute error 5.31).

Conclusions: Automatic speech analysis could help with early detection of subtle signs of stress in vulnerable populations over the phone. By combining the use of this technology with timely intervention strategies, it could contribute to the prevention of burnout and the development of comorbidities, such as depression or anxiety.

(J Med Internet Res 2021;23(4):e24191) doi: 10.2196/24191

\section{KEYWORDS}

stress detection; speech; voice analysis; COVID-19; phone monitoring; computer linguistics 


\section{Introduction}

In December 2019 in the Chinese city of Wuhan, a new coronavirus pneumonia, COVID-19, emerged. The pathogen involved is SARS-CoV-2. Here, we will refer to the pathology as COVID-19. COVID-19 has spread very rapidly in China but also in many other countries [1]. On March 11, 2020, the World Health Organization declared that the COVID-19 outbreak had become a pandemic [2].

According to previous studies on SARS or Ebola epidemics, the onset of a sudden and immediately fatal disease could put extraordinary pressure on health care professionals [3]. Increased workloads, physical exhaustion, inadequate personal equipment, nosocomial transmission, and the need to make ethically difficult decisions about rationing care can have dramatic effects on their physical and mental well-being. Their resilience may be further compromised by isolation and loss of social support, risk or loss of friends and relatives, and radical, often worrying changes in working methods. Health care workers are, therefore, particularly vulnerable to mental health problems, including fear, anxiety, depression, and insomnia [4,5]. Initial results estimate that $23 \%$ and $22 \%$ of health care workers experienced depression and anxiety, respectively, during the COVID-19 pandemic [6].

Paradoxically, health care workers do not tend to seek professional help, and stress-related symptoms are often not immediately reported: "burnout, stress, and anxiety will have to wait." Most of the time there will not even be a demand for care. Early implicit stress detection is of great importance in this population and would allow for timely intervention strategies in order to prevent escalation and complete occupational burnout.

To measure stress in clinical practice, various scales and questionnaires are available, such as the Perceived Stress Scale (PSS) [7], the Stressful Life Event Questionnaire [8], the Stress Overload Scale [9], and the Trier Inventory for Chronic Stress [10]. However, the present health crisis pushed research teams to investigate the use of new technological tools in this specific population. One possible avenue is the use of automatic speech analysis allowing extraction of voice features during standard consultation or over a simple phone call.

Psychological stress induces multiple effects on the body, including increased muscle tension, increased breathing rate, and changes in salivation rate, which may, in turn, affect vocal production $[11,12]$. Under psychological stress, voice pitch (ie, the acoustic correlate of fundamental frequency [F0]) usually increases, as it is inversely related to the rate of vocal fold vibration, which stretches under stress and becomes tenser together with an increase in subglottal pressure and vocal intensity $[13,14]$. Indeed, an increase in voice pitch is the most commonly reported finding in studies examining speech under stress. However, stress can also affect other voice parameters, such as an increase in speech prosody $[11,13]$. In depression, the analysis of speech characteristics has recently attracted considerable research attention [15-17]. Studies revealed that patients show flattened affect, reduced speech variability, monotonicity in pitch and loudness, increased pause duration, and reduced speech rate [18-20]. A recent study investigated the use of speech parameters extracted from audio recordings to differentiate patients suffering from posttraumatic stress disorder from healthy controls [21].

Thus, the detection of subtle events in the voice may offer a window into assessing the impact of stress in situations where circumstances make it difficult to monitor stress directly but need to be addressed urgently [22].

In this work, we aim to investigate the use of a rapid and remote measure of stress levels in health professionals working during the COVID-19 outbreak, utilizing the automatic analysis of their speech behavior during a short phone call conversation.

Firstly, speech samples of health care professionals were collected over the phone during the COVID-19 pandemic, and various voice features were extracted and compared with classical stress measures. Secondly, based on the extracted features, scores from the completed stress scale that were obtained by participants were predicted. The purpose of this pilot study was to assess whether this technological method could be of interest to support early screening of subtle signs of stress.

\section{Methods}

\section{Participants}

Health care professionals were recruited through outreach telephone calls. They worked during the COVID-19 outbreak in the local university hospital center of Nice, France, in either private practices or as independent workers in the Provence-Alpes-Côte d'Azur region. They could occupy any function in these structures. The only criterion for noninclusion was the subjects' refusal to participate in the study. Inclusion of participants was carried out from May 5 to June 7, 2020.

The study was approved by the Ethical Board for noninterventional studies of the University Côte d'Azur, France (approval 2020-58). Participants were given all the information about the study prior to the call so they could give informed consent. For those interested, the option for a follow-up call with a clinician was provided.

\section{Procedure}

The telephone calls were made by psychiatrists $(n=3)$ or psychologists $(n=1)$ belonging to the Cognition Behavior Technology research team and the memory clinic of the University Côte d'Azur. Calls lasted about 15 minutes and were composed of the following:

1. An informative part explaining the reasons for the call and its structure and how the study is conducted. The participant's consent was requested to continue and to proceed with a recording of his or her voice.

2. The Motivation Stress Affect (MSA) questionnaire. The MSA questionnaire is a self-administered questionnaire composed of 11 questions that must be answered by "yes" or "no." The first five questions assess motivation [23], the next two questions assess depression, and the last four questions assess stress [24]. 
3. Three open standardized questions: neutral, positive, and negative storytelling. In order to capture natural speech, but within a limited time frame, the participant was asked to talk about something emotionally neutral (ie, describe where he or she is), to talk about a negative event in his or her life, and, finally, to talk about a positive event in his or her life. Each answer should have lasted about 1 minute and was recorded in a secure and encrypted way. It was not specified whether the event had to be experienced during COVID-19; thus, it was open to the participant to recall whatever event first came to mind. These free-speech tasks were used in previous studies [18,25] and allowed for a greater range of induced emotional effects, potentially sensitive to signs of stress and depression. The comparison of speech features between neutral and emotionally loaded questions may give insight into the affective state of participants.

4. The PSS. This scale [7] is a hetero-questionnaire composed of 10 questions to be answered by "never," "almost never," "sometimes," "quite often," or "often."

5. An open listening part aimed at exploring certain points in greater depth in order to refine the clinical needs.

6. Decision and advice. Following the above steps, the psychiatrist or psychologist offered or did not offer psychological follow-up depending on whether he or she considered that the patient was at risk of developing or had a mood or anxiety disorder. He or she may also have offered advice on intervention strategies (eg, relaxation, yoga, physical activity, and national call platform for psychological support for caregivers).

\section{Materials}

To perform the phone calls for this study, the phone version of the DELTA application [26] was used. The DELTA solution allows for the use of a dedicated interface in the form of an iOS app to make phone calls and locally record these calls on the internal memory of an iPad. The phone calls were made directly with the iPad and through its internal microphone.

These recordings were then automatically transmitted - the iPad had to be connected to the internet-to the DELTA application programming interface (API) for analysis of acoustic and semantic parameters. Once the analysis was complete, the results were displayed directly on the DELTA interface. The recordings were made locally on the phone, the connection between the interface and the DELTA API was secure and encrypted, and the recordings were destroyed from the DELTA servers once the analysis was complete and the results sent to the experimenter.

\section{Analysis}

Audio features were extracted directly and automatically from the recorded audio signals of the three open standardized questions (see item \#3 in the Procedure section). Characteristics were extracted from four main areas:

1. Prosodic characteristics, on long-term variations in perceived stress and speech rhythm. Prosodic features also measure alterations in personal speech style (eg, perceived pitch and speech intonation).
2. Formant characteristics represent the dominant components of the speech spectrum and convey information about the acoustic resonance of the vocal tract and its use. These markers are often indicative of articulatory coordination problems in motor speech control disorders.

3. Source characteristics that are related to the source of voice production, the airflow through the glottal speech production system. These features make operational irregularities in the movement of the vocal fold (eg, voice quality measurements).

4. Temporal characteristics include measures of the proportion of speech (eg, duration of pauses and duration of speech segments), speech segment connectivity, and overall speech rate.

Features were extracted using Python 3.7 (Python Software Foundation) [27] and free and publicly available packages. For the temporal features, the My-Voice Analysis [28] package was used. This package was built off of the speech analysis research tool praat [29]. Temporal features were actualized as the speech rate, syllable count, rate of articulation, speaking duration, total duration, and ratio of speaking to nonspeaking. This package was also used to extract prosodic features, namely the F0 values: mean, standard deviation, minimum, maximum, and upper and lower quartiles. The F0 value is the representation of what is known as the pitch.

Formant features were calculated using the Python Speech Features library [30]. To characterize this aspect of speech, the original sound recording was refit according to a series of transformations commonly used for speech recognition that yield a better representation of the sound called the mel-frequency cepstrum (MFC). From this new representation of the sound form, the first 14 coefficients of the MFC were extracted. The MFC values were extracted given that they describe the spectral shape of the audio file, generally with diminishing returns in terms of how informative they are, which is why we only considered the first 14 coefficients. If we were to select a greater number of MFC values, it would result in a potentially needlessly more complex machine learning model using less informative features.

From each of these waves, the mean, variance, skewness, and kurtosis were calculated for the energy (static coefficient), velocity (first differential), and acceleration (second differential).

The Librosa package [31] was used to calculate the mean, maximum, minimum, and standard deviation of the root mean square value, centroid, bandwidth, flatness, zero-crossing rate, loudness, and flux of the spectrogram, or the visualization of the recording.

The source characteristics were extracted using the Signal_Analysis package, version 0.1 .26 , to extract the micromovements of the sound wave: harmonics-to-noise ratio (HNR), jitter, shimmer, and glottal pulses. Jitter and shimmer are two features of vocal signals that describe the frequency variation from cycle to cycle of the sound wave and the waveform amplitude, respectively [32,33]. While jitter rises with the growing lack of control of vocal cord vibration, higher shimmer is coupled with increased breathiness. HNR is the ratio between periodic components and nonperiodic components that 
constitute a voiced speech segment [34]. These components correspond to the vibration from vocal cords and glottal noise, respectively.

Speech features vary naturally between males and females due to differences in the length of the vocal tract. These differences have been leveraged in gender classification through speech analysis based on pitch and formant frequencies [35], HNR [36], linear predictive components, and mel-frequency cepstral coefficients (MFCCs) [37]. Previous work found differences in speech depending on gender in the effects of depression and the effectiveness of classifiers for its detection [38]. This is why this study considers males and females separately.

\section{Statistical Analysis}

The data collected were described using mean and standard deviation for quantitative variables, and frequency and percentage for qualitative variables. Demographic characteristics, such as age and gender, were compared between different groups of caregivers using a chi-square test for qualitative variables (eg, gender) and an analysis of variance performed for quantitative variables (eg, age). Similarly, the data measured for voice and scores were compared between different groups of caregivers. The normality of the collected data was tested using a Shapiro test. In order to test the relationship between the different voice measures and the measured scores, Spearman correlations were used. In addition, to test the link between the voice measures and the therapist's decision, Student $t$ tests or Wilcoxon-Mann-Whitney tests were performed. A $P$ value of less than .05 was considered significant The analyses were performed using the free statistical software RStudio 4.0.0 [39]. Further, regression analyses were performed with the extracted vocal features to determine the error rate for predicting the participants' stress scores.

\section{Results}

\section{Participants}

In total, 89 French-speaking health professionals, aged between 20 and 74 years, accepted the outreach phone calls and their speech samples were recorded and analyzed. Their demographic characteristics are presented in Table 1.

The mean age of the participants was 40.53 years (SD 14.19). The mean stress score on the PSS was 22.43 (SD 7.16) and on the MSA questionnaire was 2.92 (SD 2.09). The majority of the participants scored below 26 on the PSS but above 0 on the MSA questionnaire. Results on the PSS and on the MSA stress scale were proportional. We found that $27 \%$ (24/89) of the recorded health professionals experienced intense stress, and $28 \%(25 / 89)$ experienced occasional stress. Only 16\% (14/89) of the participants requested a follow-up. The stress level was gender dependent, with females reporting higher stress levels. For males, stress levels tended to drop with age. Figure 1 shows a distribution of the total stress scores across genders. The total stress scores in the female group are more dispersed than in the male group and are generally higher. A total of 14 out of 88 (16\%) participants $(11 / 57,19 \%$ of all females; $3 / 31,10 \%$ of all males) asked for a follow-up call. Their mean PSS score (mean 31.78, SD 7.40) and mean MSA scale score (mean 5.57, SD 1.34) were significantly higher than for those who did not ask for a follow-up, whose mean PSS score was 20.60 (SD 5.63) and mean MSA scale score was 2.38 (SD 1.8). 
Table 1. Descriptive statistics for participant characteristics $(\mathrm{N}=89)$.

\begin{tabular}{|c|c|c|c|c|}
\hline \multirow[t]{2}{*}{ Characteristic } & \multicolumn{3}{|c|}{ Participants, n (\%) } & \multirow[t]{2}{*}{$P$ value $^{\mathrm{a}}$} \\
\hline & Total $(\mathrm{N}=89)$ & Male $(n=31)$ & Female $(\mathrm{n}=58)$ & \\
\hline Gender & $89(100)$ & $31(35)$ & $58(65)$ & $N / A^{b}$ \\
\hline Education (years) $(\mathrm{n}=81)$ & & & & .03 \\
\hline$<12$ & $19(23)$ & $1 / 28(4)$ & $18 / 53(34)$ & \\
\hline$\geq 12$ & $62(77)$ & $27 / 28(96)$ & $35 / 53(66)$ & \\
\hline Timing of call & & & & .03 \\
\hline During lockdown & $34(38)$ & $7(23)$ & $27(47)$ & \\
\hline After lockdown & $55(62)$ & $24(77)$ & $31(53)$ & \\
\hline Perceived Stress Scale score & & & & .47 \\
\hline Knows how to manage stress $(<21)$ & $40(45)$ & $16(52)$ & $24(41)$ & \\
\hline Generally knows how to cope with stress (21-26) & $25(28)$ & $9(29)$ & $16(28)$ & \\
\hline Life is a constant threat $(>26)$ & $24(27)$ & $6(19)$ & $18(31)$ & \\
\hline Motivation Stress Affect (MSA) scale score & & & & .99 \\
\hline 0 & $23(26)$ & $8(26)$ & $15(26)$ & \\
\hline$>0$ & $66(74)$ & $23(74)$ & $43(74)$ & \\
\hline MSA motivation scale score & & & & .47 \\
\hline 0 & $30(34)$ & $12(39)$ & $18(31)$ & \\
\hline$>0$ & $59(66)$ & $19(61)$ & $40(69)$ & \\
\hline MSA depression scale score & & & & .32 \\
\hline 0 & $57(64)$ & $22(71)$ & $35(60)$ & \\
\hline$>0$ & $32(36)$ & $9(29)$ & $23(40)$ & \\
\hline Follow-up request (n=88) & & & & .36 \\
\hline No & $74(84)$ & $28(90)$ & $46 / 57(81)$ & \\
\hline Yes & $14(16)$ & $3(10)$ & $11 / 57(19)$ & \\
\hline
\end{tabular}

${ }^{\mathrm{a}}$ Chi-square test or Fisher exact test.

${ }^{\mathrm{b}} \mathrm{N} / \mathrm{A}$ : not applicable; the $P$ value was not calculated for gender.

Figure 1. Stress score distribution across genders.

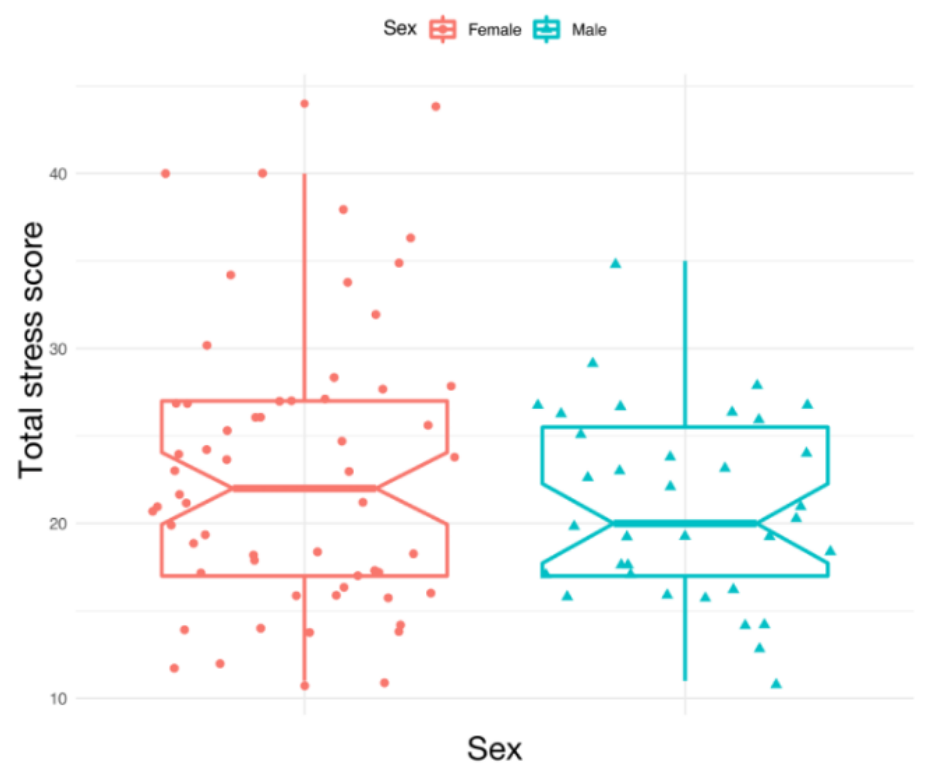




\section{Correlations}

First, vocal and nonvocal features were analyzed in relation to the stress level. The data set was quite small and, therefore, rather than training a classifier, we performed correlation analysis between the features computed for each speech task and the reported stress level. Further, only extracted speech features were considered; a priori, nonmeaningful features, like ID, were removed.

We performed a selection of the top $\mathrm{k}$ features based on their descriptive power for the target variable total stress score. Vocal features might be gender dependent. Therefore, we performed a selection of top features for male and female data sets separately. We used Spearman correlation, since we had both ordinal and continuous features: the target total stress score is ordinal. Since Spearman correlation uses only the ranks of the variables and not their raw values, we could omit the normalization step. We considered absolute values of the correlation coefficient for feature scoring. Results are presented in Table 2.

The main speech parameters correlating with stress levels in both genders were spectral (ie, formant) features, namely the MFCCs. These features characterize the spectrum of speech, which is the frequency distribution of the speech signal at a specific time. MFCCs were derived by computing a spectrum of the log-magnitude mel-spectrum of the audio segment. The lower coefficients represent the vocal tract filter and the higher coefficients represent periodic vocal fold sources [18]. Moreover, in males' prosodic characteristics, such as the F0, and in females with the positive storytelling, pitch ranges were associated with stress levels.

Table 2. Correlation between stress levels and speech features.

\begin{tabular}{|c|c|c|}
\hline Top 10 features for each data set & Task & Spearman correlation \\
\hline \multicolumn{3}{|l|}{ Female data set } \\
\hline MFCC $^{\mathrm{a}} 3$ acceleration skewness & Positive story & 0.49 \\
\hline MFCC2 mean & Neutral story & 0.44 \\
\hline Pitch range & Positive story & 0.44 \\
\hline MFCC3 acceleration skewness & Negative story & 0.43 \\
\hline MFCC2 mean & Positive story & 0.44 \\
\hline MFCC5 acceleration kurtosis & Negative story & -0.42 \\
\hline MFCC2 mean & Negative story & 0.43 \\
\hline MFCC5 velocity kurtosis & Negative story & -0.40 \\
\hline MFCC3 acceleration skewness & Neutral story & 0.39 \\
\hline MFCC5 velocity kurtosis & Negative story & 0.39 \\
\hline \multicolumn{3}{|l|}{ Male data set } \\
\hline Upper quartile $\mathrm{F}^{\mathrm{b}}$ & Neutral story & -0.54 \\
\hline Pronunciation posteriori probability score percentage & Positive story & -0.50 \\
\hline Energy acceleration mean & Positive story & 0.52 \\
\hline Mean F0 & Neutral story & -0.51 \\
\hline MFCC9 kurtosis & Positive story & 0.41 \\
\hline MFCC9 variance & Positive story & -0.44 \\
\hline Upper quartile F0 & Negative story & -0.47 \\
\hline MFCC4 acceleration mean & Positive story & -0.40 \\
\hline Upper quartile F0 & Positive story & -0.47 \\
\hline MFCC12 acceleration skewness & Neutral story & -0.42 \\
\hline
\end{tabular}

${ }^{a}$ MFCC: mel-frequency cepstral coefficient; the numbers following MFCC are part of the feature names presenting their location on a spectrum.

${ }^{\mathrm{b}} \mathrm{F} 0$ : fundamental frequency.

For female participants, correlation analyses between negative, positive, and neutral features and the target feature total stress score were performed. Among the top 5 features, we have MFCC acceleration skewness, which correlates with the stress level by 0.45 and 0.37 in the positive and neutral tasks, respectively. The other features among top 5 features are task specific. Thus, for each task there is a different set of features associated with stress level.

For male participants, the selection was performed analogously. The top features are task specific as well, and they differ from the features for the female data set. In this sample, we obtained more negatively correlating features than for the female data 
set; this meant that features, for instance, related to $\mathrm{F} 0$ of low value (mean F0 in the neutral story with -0.51 , upper quartile F0 in the negative and positive story with -0.47 , and upper quartile F0 in the neutral story with -0.54) are associated with high stress scores. In general, low values represent a smaller pitch range.

\section{Regression}

Stress scores were regressed against measurements for positive, neutral, and negative tasks. Similarly, the regression for tasks of different sentiments was performed for groups of female and male participants to allow for possible impacts of gender on stress levels. For the regressors, we used linear, support vector machine (SVM), and random forest regressors to predict the stress scores.

The first regression approximated the stress score by estimating coefficients for each feature in the training data, where greater coefficients indicate a greater influence over the predicted value. Linear regression models are fast, highly interpretable, and commonly used for prediction of stress scores from audio features and speech analysis, according to previous studies [40-42]. The random forest regressor created a number of decision trees that were constructed based on random sampling from the training data; each tree then attempted to determine the best way to predict the scores given the data it received. Each decision tree outputted a predicted value and the mode value was selected. Decision tree methods have shown high accuracy with good interpretability in similar studies where vocal and linguistic features were employed for detection of emotions, social signals, and mental health problems [43-45]. The SVM regressor took each set of features and projected them as a vector onto a space and attempted to find the optimal way to separate the data. The stress score was then based on the distance from that separator. Stress modeling with inputs from physiological sensors or audio sources using SVM has also been previously reported to give high model performance [46-48]. In recent studies, both SVM and random forest provided notably high prediction and classification strength for stress detection using various speech features [49-51].

The caret package from R, version 3.4.2 (The R Foundation), was used for data training and validation. A 10-fold cross-validation was performed and performance was evaluated using the mean absolute error (MAE): the average of the absolute difference between the predicted and actual values from our models for all participants. The score ranges from 0 to infinity, where a score closer to 0 indicates a better-fitting model.

The prediction of total stress scores using all or a subset of tasks among male or female subjects was carried out using various baseline regression models, whose performances were evaluated by the plots in Figure 2, where the MAE values are presented on the y-axis. Overall, the prediction strength in males was better than in females for all sentiments, as shown by a trend of lower errors (lowest MAE for males was 3.84; lowest MAE for females was 5.56). It is notable that stress score regression models based on negative tasks in males and neutral tasks in females performed relatively poorly compared to other tasks. For both male and female participants, using positive tasks for regression yielded equivalent or better results than using all tasks, suggesting that a subset of tasks could be employed for accurate and less time-consuming prediction of stress scores. An overview of the lowest scores for each testing scenario is presented in Table 3.

All regression models outperformed their respective baseline MAE values (4.46 and 6.35 in males and females, respectively). Linear models and the SVM regressor were the most precise for the prediction of total stress scores in general. 
Figure 2. Performances of different computerized regression models in predicting stress levels based on vocal features. Boosted: boosted linear model; ElasticNet: mix of L1 and L2 regularized linear regression; MAE: mean absolute error; Poly: support vector machine with polynomial basis function kernel; Quantile: quantile regression forest; Radial: support vector machine with radial basis function kernel; SVM: support vector machine.

\section{Regressor family: $\rightarrow$ - Linear $\rightarrow$ Random Forest $\rightarrow$ SVM}
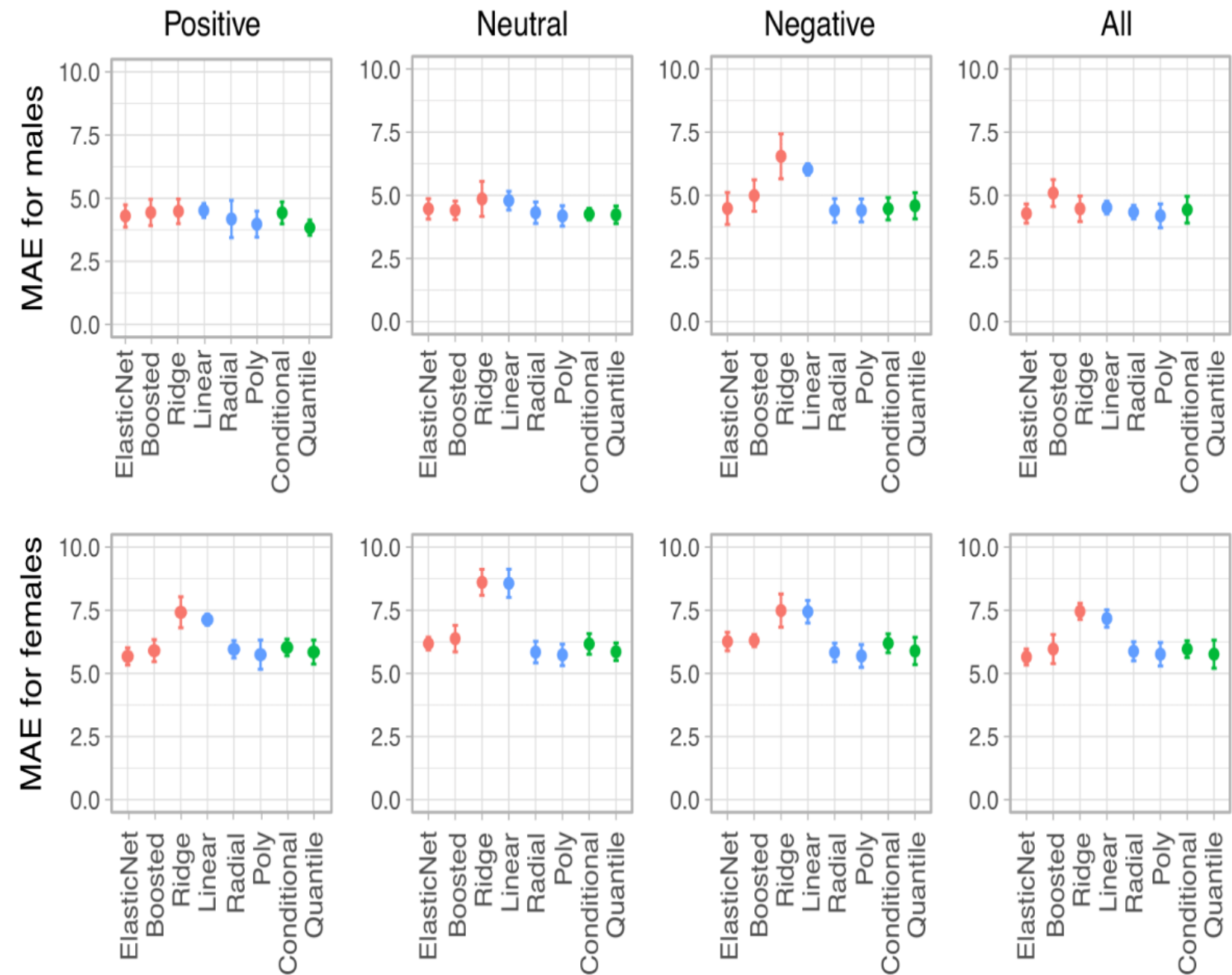

Table 3. The lowest scores for each testing scenario.

\begin{tabular}{|c|c|c|c|c|c|c|}
\hline \multirow{2}{*}{$\begin{array}{l}\text { Participant } \\
\text { group }\end{array}$} & \multicolumn{2}{|l|}{ Positive tasks } & \multicolumn{2}{|c|}{ Neutral tasks } & \multicolumn{2}{|c|}{ Negative tasks } \\
\hline & $\mathrm{MAE}^{\mathrm{a}}(\mathrm{SD})$ & Model & MAE (SD) & Model & MAE (SD) & Model \\
\hline All & $5.31(0.25)$ & ElasticNet $^{b}$ & $5.25(0.28)$ & QuantileRF $^{\mathrm{c}}$ & $5.34(0.35)$ & PolySVM $^{\mathrm{d}}$ \\
\hline Male & $3.84(0.43)$ & QuantileRF & $4.40(0.37)$ & BoostedLM $^{\mathrm{e}}$ & $4.37(0.43)$ & PolySVM \\
\hline Female & $5.56(0.41)$ & ElasticNet & $5.84(0.42)$ & RadialSVM $^{\mathrm{f}}$ & $5.68(0.45)$ & PolySVM \\
\hline
\end{tabular}

${ }^{\mathrm{a}} \mathrm{MAE}$ : mean absolute error.

${ }^{b}$ ElasticNet: mix of L1 and L2 regularized linear regression.

${ }^{\mathrm{c}}$ QuantileRF: quantile regression forest.

${ }^{\mathrm{d}}$ PolySVM: support vector machine with polynomial basis function kernel.

e BoostedLM: boosted linear model.

${ }^{\mathrm{f}}$ RadialSVM: support vector machine with radial basis function kernel. 


\section{Discussion}

\section{Principal Findings}

The purpose of this study was to investigate the potential of using automatic speech analysis for the detection of stress in health care professionals during the current COVID-19 pandemic. This would potentially lead to earlier and timely prevention among this high-risk population. Firstly, speech samples were collected over the phone, and various voice features were extracted and compared with classical stress measures. Secondly, based on the extracted features, scores obtained by participants on the completed stress scale were predicted.

The main outcome of this study was the demonstration of this approach's feasibility under the given context, as all participants were cooperative and appreciated the initiative of rapidly applying this existing technology to this specific use case. Moreover, from phone call recordings, a number of vocal correlates of stress have been identified, namely in the area of spectral features (ie, MFCC) as well as prosodic features such as F0, which seem to be the most commonly reported features in well-controlled trials [11]. Stress scores could be predicted based on speech features with relatively small errors.

Spectral features characterize the speech spectrum; the frequency distribution of the speech signal at a specific time indicates information in some high-dimensional representation [18]. The features capture information regarding changes in muscle tension and control and have consistently been observed to change with a speaker's mental state. A few depression studies reported a relative shift in energy with increasing depression severity $[52,53]$.

Another result we obtained was that most identified vocal features were task dependent as well as gender dependent. Interestingly, in the female group, MFCC features seemed to be associated with stress levels during all tasks, meaning that it did not matter what participants were talking about; as long as sufficient speech was captured, meaningful information could be extracted and subtle signs of stress level could be detected. On the other hand, in the male data set, the upper quartile F0 appeared as a task-independent feature sensitive to stress levels. Overall, in the male data set, we observed more features with a negative correlation than we did for the female data set.

Voice production can be divided into three processes: breathing, phonation, and resonance stress [54]. For the second process, phonation, the vocal folds must close and open again to create vibration. The frequency rate of these pulses determines the F0 of the vocal source contributing to the perceived pitch of the sound.

Previous research showed that increased muscle tension tends to be caused by stress $[55,56]$, resulting in a tensing of the vocal folds, which, in turn, most likely causes a raising of F0. A recent review on voice analysis in stress [22] stated that the parameter F0 has been considered as a "universal stress indicator," whereas increased levels of F0 might be linked with acute bottom-up processes of sympathetic arousal. Similar studies of analysis of phone call recordings during situational stress situations revealed an increase in F0 and intensity with presumed levels of stress $[55,57,58]$. Our findings seem consistent with the majority of acoustic studies, pointing to F0 as one important marker of stress levels.

However, most correlations we found were with resonance (ie, formant) parameters, which are involved in the quality of sound shaping and vowel and consonant pronunciation and are produced by the muscle activity involved in the shaping of the resonant cavities of the vocal tract system [59]. These parameters are less documented in regard to stress. The MFCC, in particular, can be indicative of breathiness in the voice [60]. Interestingly, one study found a circadian pattern in MFCCs due to sleep deprivation. For this, voice perturbations were compared with classical sleep measures [61] and correlations were found between fatigue scores and MFCCs. This might eventually explain our results, as most participants also reported signs of fatigue during the interviews.

Another study examined speech in students under exam stress and a few days later; in this case, heart rate was measured to control for the actual stress levels. Under stress, students' heart rates increased, F0 and F0 SD increased, first formant (F1) and second formant (F2) frequencies increased, and MFCCs decreased in relation to baseline levels [62].

It can be hypothesized that given our recorded population who reported relatively mild to moderate levels of stress, rather subtle changes in voice parameters were found and, therefore, weaker correlations were observed. However, it is important to underline that changes in features that we found to be sensitive to stress levels were gender dependent but not necessarily task dependent. They were most likely too small to be detectable by the human ear but were captured by the automatic speech analysis. We assume that by applying this technology to regular check-up calls with people experiencing high stress levels, such as health care professionals, very early signs of stress can be detected in their voices, allowing for timely preventive strategies.

Regression models using vocal features performed relatively well in predicting stress scores, namely in the positive story task for both genders (MAE of 5.31). It shows that the technology could capture indicative patterns from even a short amount of time, possibly even from one task, to recognize tendencies of stress levels in a fragile but healthy population; this represents a promising rapid tool for prediction of stress scores.

\section{Strengths of This Study}

This study is a first step into the early identification of stress in an at-risk population, such as caregivers, who do not directly express their psychological suffering. We can imagine extending this technique to other fragile populations for early screening of stress, such as teenagers who are victims of school harassment or women who are victims of abuse, where timely management could potentially prevent the development of comorbidities, such as depression and anxiety. Moreover, patient populations who have difficulty expressing their problems, such as those with autism spectrum disorder or dementia, could benefit from this technology. 
Generally, remote psychological counseling is controversial. Nevertheless, it is becoming necessary due to current economic, social, and health constraints, but has been received by professionals and patients with mixed feelings. Indeed, the nonverbal part of communication is lost and the dynamics of interaction are not the same. However, contrary to these preconceived ideas, we have noticed during this work that it is easier for certain participants to open up and speak about personal issues during these interviews in a liberating manner, similar to a confessional. Not being in the physical presence of the listener may facilitate personal expression, with less fear of being judged. This aspect is very interesting during a screening because it considerably accelerates the process of detection and diagnosing of psychological symptoms.

\section{Weaknesses of the Study}

This project has been rapidly implemented, initially with an approach of qualitative and quantitative data analysis, that should contribute to the early and timely assistance of health professionals during the COVID-19 pandemic. The staff members available to participate in the study were limited. Patient selection was done on a voluntary basis. It is conceivable that the population studied were more concerned about their state of psychological suffering and, therefore, potentially had a selection bias.

Although the voice recordings were made in the middle of the interview without this time being precisely stated, it is possible that some patients may have suspected this, which could have been anxiety provoking and skewed our results. Recording throughout the interview for parameters not affected by the tasks would provide more data and more robust results.
Finally, the obtained correlations can be considered as rather moderate, which makes it difficult to draw any strong conclusions. A larger data set, ideally of a longitudinal nature, with more precise characterization of the speakers is needed in order to verify whether the correlating features represent real markers of stress.

\section{Future Perspective}

For future work, we propose to perform this analysis on a larger data set and to build a prediction model. In case of an insufficient number of observations per stress level, the number of stress levels can be reduced by binning. Binning can also be carried out on characteristic values.

Further studies with acoustic measurements and stress questionnaires at regular time intervals would allow for the analysis of the kinetics of the markers and a better perception of their sensitivity and specificity. In addition, adding clinical measurements of psychiatric symptoms, such as the Diagnostic and Statistical Manual of Mental Disorders, Fifth Edition [63], would make it possible to perceive whether one of the markers is predictive of an anxiety or depression disorder. The use of the tool could be combined with the delivery of preventive strategies, such as physical exercises, adaptation of diet, psychotherapy, meditation, or the use of symptomatic treatments, and it could be employed at the same time for the evaluation of the obtained effects. However, in order to produce a real-world application of this technology, larger validation studies have to be performed to demonstrate clinical meaningfulness by comparing its performance to standardized measurement tools.

\section{Acknowledgments}

This research was supported by a grant from the Fondation GSF (Groupe Services France) Jean Louis Noisiez and the Association Innovation Alzheimer. Thanks to all of the professional health care workers who agreed to participate in this study.

\section{Authors' Contributions}

AK, KR, JE, and PR designed and conducted the study. AD contributed as technical support to this study. NL, HL, and RF analyzed the data. KR, AK, NL, HL, and PR drafted the manuscript. All authors have read and agreed to the published version of the manuscript.

\section{Conflicts of Interest}

$\mathrm{NL}$ is an employee and shareholder of ki elements UG.

\section{References}

1. Pneumonia of unknown cause - China. World Health Organization. 2020 Jan 05. URL: https://www.who.int/csr/don/ 05-january-2020-pneumonia-of-unkown-cause-china/en/ [accessed 2020-01-05]

2. WHO announces COVID-19 outbreak a pandemic. World Health Organization. 2020 Mar 12. URL: https://www.euro.who.int/ en/health-topics/health-emergencies/coronavirus-covid-19/news/news/2020/3/who-announces-covid-19-outbreak-a-pandemic [accessed 2020-03-12]

3. Liu X, Kakade M, Fuller CJ, Fan B, Fang Y, Kong J, et al. Depression after exposure to stressful events: Lessons learned from the severe acute respiratory syndrome epidemic. Compr Psychiatry 2012 Jan;53(1):15-23 [FREE Full text] [doi: 10.1016/j.comppsych.2011.02.003] [Medline: 21489421]

4. Lung F, Lu Y, Chang Y, Shu B. Mental symptoms in different health professionals during the SARS attack: A follow-up study. Psychiatr Q 2009 Jun;80(2):107-116. [doi: 10.1007/s11126-009-9095-5] [Medline: 19247834] 
5. Wu P, Fang Y, Guan Z, Fan B, Kong J, Yao Z, et al. The psychological impact of the SARS epidemic on hospital employees in China: Exposure, risk perception, and altruistic acceptance of risk. Can J Psychiatry 2009 May;54(5):302-311 [FREE Full text] [doi: 10.1177/070674370905400504] [Medline: 19497162$]$

6. Pappa S, Ntella V, Giannakas T, Giannakoulis VG, Papoutsi E, Katsaounou P. Prevalence of depression, anxiety, and insomnia among healthcare workers during the COVID-19 pandemic: A systematic review and meta-analysis. Brain Behav Immun 2020 Aug;88:901-907 [FREE Full text] [doi: 10.1016/j.bbi.2020.05.026] [Medline: 32437915]

7. Cohen S, Kamarck T, Mermelstein R. A global measure of perceived stress. J Health Soc Behav 1983 Dec;24(4):385-396. [Medline: 6668417]

8. Roohafza H, Ramezani M, Sadeghi M, Shahnam M, Zolfagari B, Sarafzadegan N. Development and validation of the stressful life event questionnaire. Int J Public Health 2011 Aug;56(4):441-448. [doi: 10.1007/s00038-011-0232-1] [Medline: $\underline{21327856]}$

9. Amirkhan J. Stress overload: A new approach to the assessment of stress. Am J Community Psychol 2012 Mar;49(1-2):55-71. [doi: 10.1007/s10464-011-9438-x] [Medline: 21538152]

10. Petrowski K, Paul S, Albani C, Brähler E. Factor structure and psychometric properties of the trier inventory for chronic stress (TICS) in a representative German sample. BMC Med Res Methodol 2012 Apr 01;12:42 [FREE Full text] [doi: 10.1186/1471-2288-12-42] [Medline: 22463771]

11. Giddens CL, Barron KW, Byrd-Craven J, Clark KF, Winter AS. Vocal indices of stress: A review. J Voice 2013 May;27(3):390.e21-390.399. [doi: 10.1016/j.jvoice.2012.12.010] [Medline: 23462686]

12. Pisanski K, Nowak J, Sorokowski P. Individual differences in cortisol stress response predict increases in voice pitch during exam stress. Physiol Behav 2016 Sep 01;163:234-238. [doi: 10.1016/j.physbeh.2016.05.018] [Medline: 27188981]

13. Kirchhübel C, Howard DM, Stedmon AW. Acoustic correlates of speech when under stress: Research, methods and future directions. Int J Speech Lang Law 2011 Sep 13;18(1):75-98. [doi: 10.1558/ijsll.v18i1.75]

14. Hollien H. Vocal fold dynamics for frequency change. J Voice 2014 Jul;28(4):395-405. [doi: 10.1016/j.jvoice.2013.12.005] [Medline: 24726331]

15. Sobin C, Sackeim HA. Psychomotor symptoms of depression. Am J Psychiatry 1997 Jan;154(1):4-17. [doi: 10.1176/ajp.154.1.4] [Medline: 8988952]

16. Schrijvers D, Hulstijn W, Sabbe BG. Psychomotor symptoms in depression: A diagnostic, pathophysiological and therapeutic tool. J Affect Disord 2008 Jul;109(1-2):1-20. [doi: 10.1016/j.jad.2007.10.019] [Medline: 18082896]

17. Bylsma LM, Morris BH, Rottenberg J. A meta-analysis of emotional reactivity in major depressive disorder. Clin Psychol Rev 2008 Apr;28(4):676-691. [doi: 10.1016/j.cpr.2007.10.001] [Medline: 18006196]

18. Cummins N, Scherer S, Krajewski J, Schnieder S, Epps J, Quatieri TF. A review of depression and suicide risk assessment using speech analysis. Speech Commun 2015 Jul;71:10-49. [doi: 10.1016/j.specom.2015.03.004]

19. Nilsonne A. Speech characteristics as indicators of depressive illness. Acta Psychiatr Scand 1988 Mar;77(3):253-263. [doi: 10.1111/j.1600-0447.1988.tb05118.x] [Medline: 3394527]

20. Leff J, Abberton E. Voice pitch measurements in schizophrenia and depression. Psychol Med 1981 Nov;11(4):849-852. [doi: 10.1017/s0033291700041349] [Medline: 7323240]

21. Marmar CR, Brown AD, Qian M, Laska E, Siegel C, Li M, et al. Speech-based markers for posttraumatic stress disorder in US veterans. Depress Anxiety 2019 Jul;36(7):607-616 [FREE Full text] [doi: 10.1002/da.22890] [Medline: $\underline{31006959]}$

22. Van Puyvelde M, Neyt X, McGlone F, Pattyn N. Voice stress analysis: A new framework for voice and effort in human performance. Front Psychol 2018;9:1994 [FREE Full text] [doi: 10.3389/fpsyg.2018.01994] [Medline: 30515113]

23. Robert P, Lanctôt KL, Agüera-Ortiz L, Aalten P, Bremond F, Defrancesco M, et al. Is it time to revise the diagnostic criteria for apathy in brain disorders? The 2018 international consensus group. Eur Psychiatry 2018 Oct;54:71-76 [FREE Full text] [doi: 10.1016/j.eurpsy.2018.07.008] [Medline: 30125783]

24. Yesavage JA, Brink T, Rose TL, Lum O, Huang V, Adey M, et al. Development and validation of a geriatric depression screening scale: A preliminary report. J Psychiatr Res 1982 Jan;17(1):37-49. [doi: 10.1016/0022-3956(82)90033-4]

25. König A, Linz N, Zeghari R, Klinge X, Tröger J, Alexandersson J, et al. Detecting apathy in older adults with cognitive disorders using automatic speech analysis. J Alzheimers Dis 2019 Jun 18;69(4):1183-1193. [doi: 10.3233/jad-181033]

26. ki:elements. URL: https://ki-elements.de [accessed 2020-11-01]

27. Van Rossum G, Drake FL. Python 3 Reference Manual. Scotts Valley, CA: CreateSpace; 2009:1-242.

28. Shahab S. My-Voice Analysis. GitHub. 2020. URL: https://github.com/Shahabks/my-voice-analysis [accessed 2020-11-01]

29. Boersma P. PRAAT, a system for doing phonetics by computer. Glot International. Vol 5, No. 9/10. 2001. URL: https:/ /www.researchgate.net/profile/Paul-Boersma-2/publication/

208032992 PRAAT a system for doing phonetics by computer/links/53d77c1c0cf29f55cfb4d26b/

PRAAT-a-system-for-doing-phonetics-by-computer.pdf [accessed 2021-04-07]

30. Lyons J. python_speech_features. GitHub. 2020 Jan 14. URL: https://github.com/jameslyons/python_speech_features [accessed 2020-11-01]

31. librosa 0.8.0. Python Package Index. 2020 Jul 21. URL: https://pypi.org/project/librosa/ [accessed 2020-11-01]

32. Kreiman J, Gerratt BR. Perception of aperiodicity in pathological voice. J Acoust Soc Am 2005 Apr;117(4 Pt 1):2201-2211. [doi: 10.1121/1.1858351] [Medline: $\underline{15898661]}$ 
33. Michaelis D, Fröhlich M, Strube HW, Kruse E, Story B, Titze IR. Some simulations concerning jitter and shimmer measurement. In: Proceedings of the 3rd International Workshop on Advances in Quantitative Laryngoscopy, Voice and Speech Research. 1998 Jun Presented at: 3rd International Workshop on Advances in Quantitative Laryngoscopy, Voice and Speech Research; 1998; Aachen, Germany p. 744-754.

34. Murphy PJ, Akande OO. Cepstrum-based estimation of the harmonics-to-noise ratio for synthesized and human voice signals. In: Proceedings of the International Conference on Nonlinear Analyses and Algorithms for Speech Processing. 2005 Apr Presented at: International Conference on Nonlinear Analyses and Algorithms for Speech Processing; April 19-22, 2005; Barcelona, Spain p. 150-160. [doi: 10.1007/11613107 13]

35. Childers D, Wu K. Gender recognition from speech. Part II: Fine analysis. J Acoust Soc Am 1991 Oct;90(4 Pt 1):1841-1856. [doi: 10.1121/1.401664] [Medline: 1755877 ]

36. Heffernan K. Evidence from HNR that/s/is a social marker of gender. Toronto Working Papers in Linguistics 2004;23:71-84 [FREE Full text]

37. Wu K, Childers DG. Gender recognition from speech. Part I: Coarse analysis. J Acoust Soc Am 1991 Oct;90(4 Pt 1):1828-1840. [doi: 10.1121/1.401663] [Medline: 1960278]

38. Low LA, Maddage MC, Lech M, Sheeber LB, Allen NB. Detection of clinical depression in adolescents' speech during family interactions. IEEE Trans Biomed Eng 2011 Mar;58(3):574-586. [doi: 10.1109/tbme.2010.2091640]

39. The Comprehensive R Archive Network. URL: https://cran.r-project.org/ [accessed 2020-11-01]

40. Gillespie S, Moore E, Laures-Gore J, Farina M, Russell S, Logan YL. Detecting stress and depression in adults with aphasia through speech analysis. In: Proceedings of the 2017 IEEE International Conference on Acoustics, Speech and Signal Processing (ICASSP). 2017 Presented at: 2017 IEEE International Conference on Acoustics, Speech and Signal Processing (ICASSP); March 5-9, 2017; New Orleans, LA p. 5140-5144. [doi: 10.1109/icassp.2017.7953136]

41. van den Broek EL, van der Sluis F, Dijkstra T. Telling the story and re-living the past: How speech analysis can reveal emotions in post-traumatic stress disorder (PTSD) patients. In: Sensing Emotions. Dordrecht, the Netherlands: Springer; 2010:153-180.

42. Muaremi A, Arnrich B, Tröster G. Towards measuring stress with smartphones and wearable devices during workday and sleep. Bionanoscience 2013;3:172-183 [FREE Full text] [doi: 10.1007/s12668-013-0089-2] [Medline: 25530929]

43. Hasan M, Rundensteiner E, Agu E. Emotex: Detecting emotions in twitter messages. In: Proceedings of the 2014 ASE Bigdata/Socialcom/Cybersecurity Conference. 2014 Presented at: 2014 ASE Bigdata/Socialcom/Cybersecurity Conference; May 27-31, 2014; San Francisco, CA p. 1-10 URL: https://web.cs.wpi.edu/ emmanuel/publications/PDFs/C30.pdf

44. Gosztolya G, Busa-Fekete R, Tóth L. Detecting autism, emotions and social signals using AdaBoost. In: Proceedings of the 14th Annual Conference of the International Speech Communication Association. 2013 Presented at: 14th Annual Conference of the International Speech Communication Association; August 25-29, 2013; Lyon, France p. $220-224$ URL: https://www.isca-speech.org/archive/archive papers/interspeech 2013/i13 0220.pdf

45. Howes C, Purver M, McCabe R. Linguistic indicators of severity and progress in online text-based therapy for depression. In: Proceedings of the Workshop on Computational Linguistics and Clinical Psychology: From Linguistic Signal to Clinical Reality. 2014 Presented at: Workshop on Computational Linguistics and Clinical Psychology: From Linguistic Signal to Clinical Reality; June 27, 2014; Baltimore, MA p. 7-16 URL: https://www.aclweb.org/anthology/W14-3202.pdf [doi: $10.3115 / \mathrm{v} 1 / \mathrm{w} 14-3202]$

46. Al-Shargie F, Tang TB, Badruddin N, Kiguchi M. Towards multilevel mental stress assessment using SVM with ECOC: An EEG approach. Med Biol Eng Comput 2018 Jan;56(1):125-136. [doi: 10.1007/s11517-017-1733-8] [Medline: 29043535]

47. Rabaoui A, Davy M, Rossignol S, Ellouze N. Using one-class SVMs and wavelets for audio surveillance. IEEE Trans Inf Forensics Secur 2008 Dec;3(4):763-775. [doi: 10.1109/tifs.2008.2008216]

48. Chang C, Chang C, Zheng J, Chung P. Physiological emotion analysis using support vector regression. Neurocomputing 2013 Dec;122:79-87. [doi: 10.1016/j.neucom.2013.02.041]

49. Soury M, Devillers L. Stress detection from audio on multiple window analysis size in a public speaking task. In: Proceedings of the 2013 Humaine Association Conference on Affective Computing and Intelligent Interaction. 2013 Presented at: 2013 Humaine Association Conference on Affective Computing and Intelligent Interaction; September 2-5, 2013; Geneva, Switzerland p. 529-533. [doi: 10.1109/acii.2013.93]

50. Sysoev M, Kos A, Pogačnik M. Noninvasive stress recognition considering the current activity. Pers Ubiquitous Comput 2015 Aug 26;19(7):1045-1052 [FREE Full text] [doi: 10.1007/s00779-015-0885-5]

51. Gjoreski M, Gjoreski H, Lutrek M, Gams M. Automatic detection of perceived stress in campus students using smartphones. In: Proceedings of the 2015 International Conference on Intelligent Environments. 2015 Presented at: 2015 International Conference on Intelligent Environments; July 15-17, 2015; Prague, Czech Republic p. 132-135. [doi: 10.1109/IE.2015.27]

52. Cummins N, Epps J, Ambikairajah E. Spectro-temporal analysis of speech affected by depression and psychomotor retardation. In: Proceedings of the 2013 IEEE International Conference on Acoustics, Speech and Signal Processing. 2013 Presented at: 2013 IEEE International Conference on Acoustics, Speech and Signal Processing; May 26-31, 2013; Vancouver, BC p. 7542-7546. [doi: 10.1109/ICASSP.2013.6639129]

53. Cummins N, Epps J, Sethu V, Breakspear M, Goecke R. Modeling spectral variability for the classification of depressed speech. In: Proceedings of the 14th Annual Conference of the International Speech Communication Association. 2013 
Presented at: 14th Annual Conference of the International Speech Communication Association; August 25-29, 2013; Lyon, France p. 857-861 URL: https://citeseerx.ist.psu.edu/viewdoc/download?doi=10.1.1.654.9966\&rep=rep1\&type=pdf

54. Kreiman J, Sidtis D. Foundations of Voice Studies: An Interdisciplinary Approach to Voice Production and Perception. Hoboken, NJ: John Wiley \& Sons; 2011.

55. Streeter L, Macdonald NH, Apple W, Krauss RM, Galotti KM. Acoustic and perceptual indicators of emotional stress. J Acoust Soc Am 1983 Apr;73(4):1354-1360. [doi: 10.1121/1.389239] [Medline: 6853847]

56. Scherer KR, Grandjean D, Johnstone T, Klasmeyer G, Bänziger T. Acoustic correlates of task load and stress. In: Proceedings of the 7th International Conference on Spoken Language Processing. 2002 Presented at: 7th International Conference on Spoken Language Processing; September 16-20, 2002; Denver, CO p. 1-4 URL: https://www.isca-speech.org/archive/ archive_papers/icslp_2002/i02_2017.pdf

57. Ruiz R, Absil E, Harmegnies B, Legros C, Poch D. Time- and spectrum-related variabilities in stressed speech under laboratory and real conditions. Speech Commun 1996 Nov;20(1-2):111-129. [doi: 10.1016/s0167-6393(96)00048-9]

58. Jessen M. Einfluss von Stress auf Sprache und Stimme: Unter besondere Berücksichtigung polizeidienstlicher Anforderungen. Idstein, Germany: Schulz-Kirchner Verlag; 2006:1-218.

59. Gopalan K, Wenndt S, Cupples EJ. An analysis of speech under stress using certain modulation features. In: Proceedings of the 25th Annual Conference of the IEEE Industrial Electronics Society. 1999 Presented at: 25th Annual Conference of the IEEE Industrial Electronics Society; November 29-December 3, 1999; San Jose, CA p. 1193-1197. [doi:

10.1109/iecon.1999.819381]

60. Hillenbrand J, Houde RA. Acoustic correlates of breathy vocal quality: Dysphonic voices and continuous speech. J Speech Hear Res 1996 Apr;39(2):311-321. [doi: 10.1044/jshr.3902.311] [Medline: $\underline{\text { 8729919] }}$

61. Greeley HP, Friets E, Wilson JP, Raghavan S, Picone J, Berg J. Detecting fatigue from voice using speech recognition. In: Proceedings of the IEEE International Symposium on Signal Processing and Information Technology. 2006 Presented at: IEEE International Symposium on Signal Processing and Information Technology; August 27-30, 2006; Vancouver, BC p. 567-571. [doi: 10.1109/ISSPIT.2006.270865]

62. Sigmund M. Introducing the database ExamStress for speech under stress. In: Proceedings of the 7th Nordic Signal Processing Symposium. 2006 Presented at: 7th Nordic Signal Processing Symposium; June 7-9, 2006; Reykjavik, Iceland p. $290-293$. [doi: 10.1109/NORSIG.2006.275258]

63. American Psychiatric Association. Diagnostic And Statistical Manual Of Mental Disorders, Fifth Edition. Arlington, VA: American Psychiatric Association Publishing; 2013.

\section{Abbreviations}

API: application programming interface

F0: fundamental frequency

F1: first formant

F2: second formant

GSF: Groupe Services France

HNR: harmonics-to-noise ratio

MAE: mean absolute error

MFC: mel-frequency cepstrum

MFCC: mel-frequency cepstral coefficient

MSA: Motivation Stress Affect

PSS: Perceived Stress Scale

SVM: support vector machine

Edited by G Eysenbach; submitted 28.09.20; peer-reviewed by A Khaleghi, S D'Arcy, E van der Velde; comments to author 24.10.20;
revised version received 13.11.20; accepted 17.03.21; published 19.04.21
Please cite as:
König A, Riviere K, Linz, N, Lindsay H, Elbaum J, Fabre R, Derreumaux A, Robert P
Measuring Stress in Health Professionals Over the Phone Using Automatic Speech Analysis During the COVID-19 Pandemic:
Observational Pilot Study
J Med Internet Res 2021;23(4):e24191
URL: $\underline{\text { https://www.jmir.org/2021/4/e24191 }}$
doi: $\underline{10.2196 / 24191}$
PMID: $\underline{33739930}$


(C)Alexandra König, Kevin Riviere, Nicklas Linz, Hali Lindsay, Julia Elbaum, Roxane Fabre, Alexandre Derreumaux, Philippe Robert. Originally published in the Journal of Medical Internet Research (https://www.jmir.org), 19.04.2021. This is an open-access article distributed under the terms of the Creative Commons Attribution License (https://creativecommons.org/licenses/by/4.0/), which permits unrestricted use, distribution, and reproduction in any medium, provided the original work, first published in the Journal of Medical Internet Research, is properly cited. The complete bibliographic information, a link to the original publication on http://www.jmir.org/, as well as this copyright and license information must be included. 